\title{
Neurosarcoidosis flare following urinary tract infection with extended spectrum beta lactamase producing Escherichia coli
}

\begin{abstract}
Neurosarcoidosis affects 5\%-10\% of Sarcoidosis patients. The etiology of a sarcoidosis flare has been unknown. Several factors have been implicated in the pathogenesis of an acute flare, including genetic, environmental and infectious agents. We report the case of a 52-year-old female with known neurosarcoidosis who presented with altered sensorium on two occasions. On both episodes she had leptomeningeal enhancements on imaging consistent with a neurosarcoidosis flare, presumably secondary to Extended Spectrum Beta Lactamase producing (ESBL) Escherichia Coli in the urine.

Keywords: ESBL, E Coli, neurosarcoidosis, status epilepticus, urinary tract infection
\end{abstract}

Volume 6 Issue 5 - 2017

\section{Ankur Sinha, Sushilkumar Satish Gupta, Ravikaran Patti, Navjot Somal}

Department of Internal Medicine, Maimonides Medical Center, USA

Correspondence: Ankur Sinha, Department of Internal Medicine, Maimonides Medical Center, USA, Tel 267-916-7785, Email ansinha@maimonidesmed.org

Received: May 13, 2017 | Published: May 17, 2017

\section{Key message}

Sarcoidosis is a disease with multifactorial causation with implications on multiple genetic, environmental and infectious agents. Literature supports the association of certain infectious agents like Mycobacterium tuberculosis, Propionibacterium acnes and Mycoplasma with a sarcoid flare.

We report an association between two acute episodes of neurosarcoidosis with ESBL E Coli infection. To the best of our knowledge this is the first ever case reported. We anticipate that this information will lead to better understanding of the etiology of the disease, and this case report will act as a stepping stone for clinicians to formulate a differential diagnosis of a flare.

\section{Introduction}

Sarcoidosis is an autoimmune disease with widespread involvement of various tissue subsets of the body. It is characterized by granulomatous inflammation with unclear etiology. Several theories have been postulated in literature, with evidence pointing towards an infectious etiology, genetic factors, environmental triggers, as well as hormonal dysregulation. ${ }^{1}$ Anecdotal data suggests that certain infections like Mycobacterium tuberculosis, Propionibacteria acne, Mycoplasma pneumoniae have been mentioned in the literature. ${ }^{2-4}$

Neurosarcoidosis affects about $5-10 \%$ of cases of sarcoidosis. ${ }^{5} \mathrm{~A}$ high percentage of cases can be missed at an initial stages, as clinical and imaging findings are often non-specific. Symptoms can be nonspecific including seizures, cranial neuropathy, and sensory or motor deficits.

We report a case where Extended Spectrum Beta Lactamase producing Escherichia Coli (ESBL E Coli) was presumed to be the trigger factor for two consecutive episodes of Neurosarcoidosis flare. To the best of our knowledge, this is the first ever case report suggesting a causal relationship between ESBL E Coli and a sarcoidosis flare.

\section{Case presentation}

52-year-old African American female presented to our facility with altered mental status. Her past medical history was significant for Neurosarcoidosis, Diabetes Mellitus, Epilepsy and Hypertension. She was febrile with a temperature of $101.6 \mathrm{~F}$ and mildly tachycardic to 103 beats per minute. Neurological examination revealed a divergent strabismus, a nonreactive right pupil, with no nuchal rigidity. Serum hematology and biochemistry was non-contributory. Urinalysis showed pyuria and bacteriuria. Chest $\mathrm{x}$-ray showed increased interstitial markings prominent in the perihilar regions. Magnetic resonance imaging(MRI) of the brain with gadolinium showed post contrast diffuse leptomeningeal enhancement on T1 images (Figure 1, panel A \& B), and there was a nodular pattern of leptomeningeal enhancement consistent with acute neurosarcoidosis (Figure 2). Lumbar puncture was unsuccessful due to body habitus. A preliminary diagnosis of urinary tract infection was formulated and broad spectrum antibiotics were instituted. Corticosteroids could not be administered as the patient was in severe sepsis.

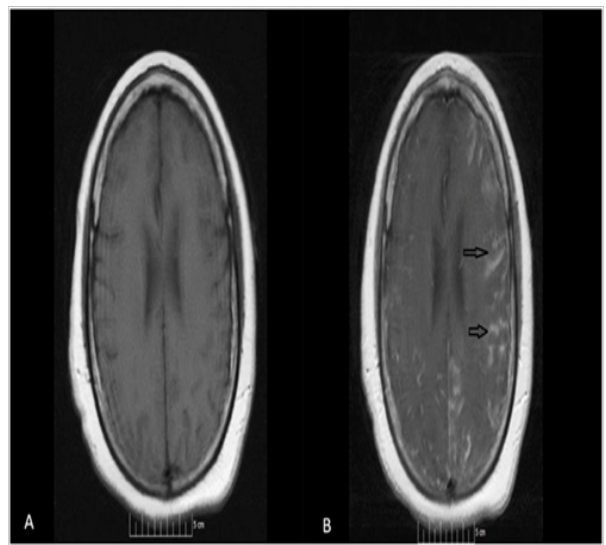

Figure I Magnetic resonance imaging (MRI) of the brain with gadolinium contrast showing post contrast diffuse leptomeningeal enhancement on $\mathrm{TI}$ images (Figure I, panel A \& B- arrows).

On day 3 of admission, the patient had a witnessed generalized tonic clonic seizure(GTCS) that was controlled by intravenous(IV) benzodiazepine, and the dose of levitracetam was titrated up to prevent further episodes. Urine culture was reported positive for ESBL $E$ Coli 
and appropriate antibiotics as per sensitivity were instituted. At this stage, the patient's white cell count improved, hemodynamics was stable. The patient was afebrile for more than 24 hours, therefore IV azathioprine and pulse dose of corticosteroids were initiated. The patient continued to have seizures despite therapy. In view of resolving sepsis, leptomeningeal enhancements seemed to be the only plausible cause for continued episodes of seizures. She was transferred to a quaternary center for continuous electroencephalogram monitoring and further management.

The patient had presented similarly in October 2012, with fulminant UTI secondary to the same microbe, followed by a Neurosarcoidosis flare. Therefore, we postulate that the ESBL E Coli infection could have acted as a trigger for the flare on both occasions.

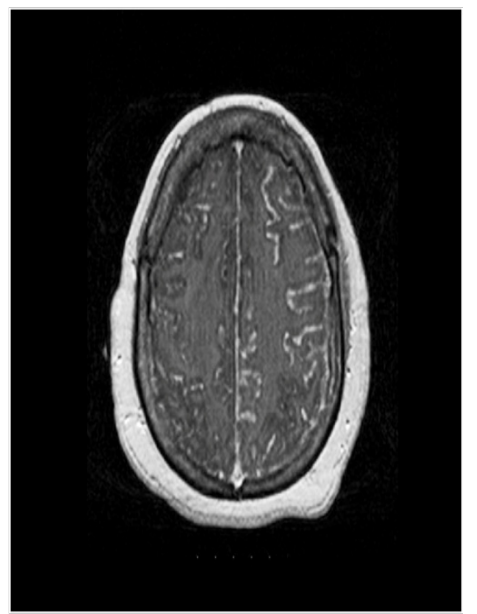

Figure $2 \mathrm{MRI}$ of the brain, TI weighted with gadolinium contrast showing a nodular pattern of leptomeningeal enhancement consistent with acute neurosarcoidosis.

\section{Discussion}

Neurosarcoidosis can have a wide spectrum of clinical presentation based on the location of the lesions. The lesions may be located at the level of the central nervous system involving the brain, meninges, and the spinal cord. It may also affect peripheral nervous systempresenting as neuropathy and myopathy.

Meningeal involvement is one of the most frequent sites of affliction. Symptom include headache, nausea, cognitive changes and gait disturbance as seen in our patient. If the involvement is diffuse, life threatening neurological emergencies like seizures, status epilepticus and hydrocephalus may ensue. Intra parenchymal lesions on the other hand may present with focal neurological deficits, endocrine dysfunction, as well as features of a space occupying lesion. Cranial nerves may also be involved with the facial nerve being the most commonly affected. ${ }^{6}$ Spinal cord involvement can lead to motor, sensory and autonomic deficits; whereas peripheral nerve involvement may present with paresthesia, radiculopathy, and focal mono-neuropathy.

Definitive diagnosis of the disease can be formulated with tissue biopsy of the affected region. Our patient had an established diagnosis of neurosarcoidosis with a confirmed tissue biopsy. Imaging studies like MRI with gadolinium is the best initial test for localization of the disease. The most common radiological finding is a leptomeningeal thickening and enhancement ${ }^{7}$ as seen in our case. CSF analysis shows elevated protein in about $54 \%$ cases. $^{8}$ An increased concentration of interleukin-2 receptors in CSF (over $150 \mathrm{pg} / \mathrm{mL}$ ) can point towards neurosarcoidosis, although the presence of concomitant infection makes this test less sensitive. ${ }^{9}$ Studies have shown that serum ACE level does not correlate well with disease activity due to low sensitivity and genetic polymorphism. ${ }^{10-11}$

The etiology of sarcoidosis continues to be an enigma. Shedding light on the basic pathology of the disease can help cement all the possible theories together. The most characteristic and consistent finding in sarcoidosis is the granuloma. It consists of epitheliod cells, and immune-histochemical analysis reveals CD4+ cells at the center of the granuloma surrounded by CD8+ cells. ${ }^{12}$ These granulomas represent the body's response to an antigen, and its attempt to wall off the injuring agent. The CD4+ cells are central to the pathogenesis of sarcoidosis, and this theory is supported by the fact that HIV infected individuals with a low CD4+ count do not mount a granulomatous response..$^{13}$ Raised levels of pro-inflammatory agents like Interleukin 2 and Tumor necrosis factor alpha have been observed in individuals with sarcoidosis and favor a TH1 response. This response can be directed towards a single etiological agent, or can be multifactorial.

An infectious etiology of sarcoidosis has been extensively studied, with Mycobacterium tuberculosis as the most investigated agent. This is supported by the fact that the mycobacterial cell wall agent tuberculostearic acid has been isolated in sarcoid granulomas. ${ }^{14}$ Other agents like Propionibacterium acnes and several viruses have been implicated as well. In order to suggest that an infectious agent led to the causation of sarcoidosis, the agent should be demonstrable in granulomatous tissue, and it should be able to reproduce a similar B and $\mathrm{T}$ cell response causing the inflammation.

In our case, consecutive episodes of ESBL $E$ Coli infection led to flares of the disease, although we were unable to obtain samples of tissue for biopsy due to inaccessible site, and risk outweighing the benefits of the procedure. Other alternative diagnoses such as occupational and environmental exposure, malignancies, and other infectious agents were considered and ruled out. IV high dose corticosteroids with immunosuppressive agents continue to be the mainstay of therapy, and were implemented. We report our findings to make clinicians and researchers aware of such an occurrence, and hope that samples for directed PCR on the granulomatous tissue may be available in future, to confirm ESBL E Coli as one of the causative agents of sarcoidosis. To the best of our knowledge this is the first ever case report highlighting the association between sarcoidosis and ESBL $E$ Coli.

\section{Acknowledgements}

None.

\section{Conflict of interest}

The author declares no conflict of interest.

\section{References}

1. Hebel R, Dubaniewicz-Wybieralska M, Dubaniewicz A. Overview of neurosarcoidosis: recent advances. J Neurol. 2015;262(2):258-267.

2. Scadding JG. Mycobacterium Tuberculosis in the Aetiology of Sarcoidosis. Bmj. 1960;2(5213):1617-1623.

3. Minami J, Eishi Y, Ishige Y, et al. Pulmonary granulomas caused experimentally in mice by a recombinant trigger-factor protein of Propionibacterium acnes. J Med Dent Sci. 2003;50(4):265-274. 
4. Jansson E, Hannuksela M. Letter: Mycoplasma antibodies in sarcoidosis. Journal of Clinical Pathology. 1974;27:510-511.

5. Judson MA, Boan AD, Lackland DT. The clinical course of sarcoidosis: presentation, diagnosis, and treatment in a large white and black cohort in the United States. Sarcoidosis Vasc Diffuse Lung Dis. 2012;29(2):119-127.

6. Stern BJ, Krumholz A, Johns C, et al. Sarcoidosis and its neurological manifestations. Arch Neurol. 1985;42(2):909-917.

7. Smith JK, Matheus MG, Castillo M. Imaging manifestations of neurosarcoidosis. AJR Am J Roentgenol. 2004;182(2):289-295.

8. Wengert O, Rothenfusser-Korber E, Vollrath B, et al. Neurosarcoidosis: correlation of cerebrospinal fluid findings with diffuse leptomeningeal gadolinium enhancement on MRI and clinical disease activity. J Neurol Sci. 2013;335(1-2):124-130.

9. Petereit HF, Reske D, Tumani H, et al. Soluble CSF interleukin 2 receptor as indicator of neurosarcoidosis. J Neurol. 2010;247:1855-1863.
10. Tomita H, Ina Y, Sugiura Y, et al. Polymorphism in the angiotensinconverting enzyme (ACE) gene and sarcoidosis. Am J Respir Crit Care Med. 1997;156(1):255-259.

11. Studdy PR, James DG. The specificity and sensitivity of serum angiotensin-converting enzyme in sarcoidosis and other diseases. In: Chretien J, et al. editors. Sarcoidosis. Paris: Pergamon Press; 1983. p. 332-344.

12. Statement on sarcoidosis. Joint Statement of the American Thoracic Society (ATS), the European Respiratory Society (ERS) and the World Association of Sarcoidosis and Other Granulomatous Disorders (WASOG) adopted by the ATS Board of Directors and by the ERS Executive Committee, February 1999. Am J Respir Crit Care Med. 1999;160(2):736755 .

13. Morris DG, Jasmer RM, Huang L, et al. Sarcoidosis following HIV infection: evidence for CD4+ lymphocyte dependence. Chest. 2003;124(3):929-935.

14. Gupta D, Agarwal R, Aggarwal AN, et al. Molecular evidence for the role of mycobacteria in sarcoidosis: a meta-analysis. Eur Respir J. 2007;30(3):508-516. 